Ferrer, R. A., Orehek, E., \& Padgett, L. S. (in press). Goal conflict when making decisions for others. Journal of Experimental Social Psychology.

Title: $\quad$ Goal Conflict When Making Decisions for Others

Authors: $\quad$ Rebecca A. Ferrer, PhD, Basic Biobehavioral and Psychological Sciences Branch, Behavioral Research Program, Division of Cancer Control and Population Sciences, National Cancer Institute, National Institutes of Health

Edward Orehek, PhD, Department of Psychology, University of Pittsburgh

Lynne S. Padgett, PhD, Strategic Direction of Hospital Systems, Cancer Control Department, American Cancer Society

Key Words: $\quad$ goal conflict; decision making; self-affirmation

Acknowledgments: There are no conflicts of interest to report. Data collected by Time-sharing Experiments for the Social Sciences, NSF Grant 0818839, Jeremy Freese and James Druckman, Principal Investigators. 


\begin{abstract}
Some of life's most important and difficult decisions are made on behalf of others. However, little is known about how goal conflict influences high-stakes decisions made on behalf of others. A nationally representative sample of U.S. healthcare providers $(n=502)$ read a statement presenting curative and palliative care goals as conflicting or complementary. We predicted and found that providers who received a goal conflict (vs. complementary) message perceived greater conflict, and rated palliative goals as less important. Providers who received a goal conflict (vs. complementarity) message also rated curative goals as less important. Moreover, there was an indirect link from goal conflict condition to willingness to provide palliative care, mediated by perceived goal conflict. A self-affirmation manipulation reduced providers' willingness to provide palliative care, but did not influence the effect of goal conflict on decision-making. Findings suggest that goal conflict is consequential for high-stakes decisions made for others, and that goal conflict (vs. complementarity) lowers importance of, and increases disengagement from, conflicting goals.
\end{abstract}




\section{Goal Conflict When Making Decisions for Others}

Some of life's most important and difficult decisions are made on behalf of others. For example, a parent may decide whether to send a child to public school or to private school, a physician may recommend palliative care and/or painful chemotherapy treatments, a psychiatrist may order a distressed patient to involuntary hospitalization or choose to continue outpatient treatment, and a health surrogate may decide whether a loved one should continue with lifesustaining treatments or institute a Do-Not Resuscitate Order. Such decisions are difficult because they involve tradeoffs with serious consequences. Emerging theoretical perspectives suggest that goal pursuit is carried out with assistance from other people: individuals help each other to pursue goals or pursue goals together (Fishbach \& Tu, 2016; Fitzsimons, Finkel, \& Vandellen, 2015; Orehek, Forest, \& Barbaro, in press). However, little is known about how people negotiate goals in the context of making high stakes decisions for others.

Understanding how individuals negotiate conflicting goals in the context of making high stakes decisions on behalf of others is important to understand because decision making on behalf of others is prevalent and consequential. Individuals often make decisions on behalf of others, particularly when others lack the capacity to make decisions for themselves (e.g., Li et al., 2018; Shah \& Raskinski, 2015; Shalowitz, Garrett-Mayer, \& Wendler, 2006; Vig et al., 2007), or when they lack the expertise to make those decisions (as may be perceived by healthcare providers who make decisions on behalf of patients rather than engaging in shared decision making; Degner \& Sloan, 1992; Gravel, \& Légaré, Graham, 2006; Murray, Pollack, White, \& Lo, 2007). Moreover, the person making the decision often experiences serious emotional repercussions as a result of the choice (Azoulay et al., 2005; Vig, Starks, Taylor, Hopley, \& Edwards, 2007; Wendler \& Rid, 2011). Indeed, decision-making sometimes comes 
with a higher emotional cost when deciding for others than when deciding for the self (ZikmundFisher, Sarr, Fagerlin, \& Ubel, 2006), especially if the decision-maker is interdependent with the other person (Polman \& Vohs, 2016). The present research aimed to better understand the influence of goal conflict on high stakes decision making for others by investigating healthcare providers' decision to provide palliative care to patients.

\section{Goal Conflict}

People have many goals, which often come into conflict with one another (Kruglanski et al., 2002; Neal, Ballard, \& Vancouver, 2017; Orehek \& Vazeou-Nieuwenhuis, 2013; Vancouver, Weinhardt, \& Schmidt, 2010). Two or more goals come into conflict when a behavioral means to one goal deters or precludes achieving the other, or because inadequate resources exist to pursue both goals (Emmons \& King, 1988; Kruglanski et al., 2013; Orehek \& Vazeou-Nieuwenhuis, 2013). At the individual decision-making level, such conflict can preclude successful pursuit of both goals, and may require a choice to pursue one goal at the expense of another (Achtziger, Gollwitzer, \& Sheeran, 2008; Goschke \& Dreisbach, 2008; Köpetz \& Orehek, 2015; Shah, Friedman, \& Kruglanski, 2002). Although in some goal conflict situations, it is possible to identify means that can facilitate both goals (Chun, Kruglanski, Sleeth-Keppler, \& Friedman, 2011; Kruglanski et al., 2013; Orehek, Forest, \& Wingrove, in press), such choices are often not readily available (Köpetz, Faber, Fishbach, \& Kruglanski, 2011). Because of this, people often pursue the more valued goal, inhibiting the activation of conflicting goals (Achtziger et al., 2008; Gollwitzer, Heckhausen, \& Stellar, 1990; Fishbach \& Zhang, 2008; Kruglanski et al., 2002; Köpetz, Kruglanski, Chen, \& Orehek, 2008) or disengaging from pursuit of those goals (Orehek, Bessarabova, Chen, \& Kruglanski, 2009; Wrosch, Scheier, Carver, \& Schulz, 2003; Wrosch, Scheier, Miller, Schulz, \& Carver, 2003). This is particularly likely when one goal is prioritized 
over the other (Chun et al., 2011; Köpetz et al., 2011; Shah et al., 2002), and can lead to motivated distortion of information in order to justify the chosen alternative (Bélanger, Kruglanski, Chen, Orehek, Johnson, 2015; Bélanger, Kruglanski, Chen, \& Orehek, 2014; Kruglanski \& Orehek, 2007). Thus, unless a choice is readily available that satisfies both currently activated, important goals, a person facing a conflict between two goals inhibits the activation of the lower priority goal, behaviorally disengages from its pursuit, and justifies their choice in favor of the higher priority goal through motivated reasoning. Despite extensive research on negotiating and resolving goal conflict at the individual decision-making level, there is a dearth of evidence on how individuals resolve goal conflict - either their own, or their perception of goal conflict experienced by the individual for whom they are making the decision - when making decisions on behalf of others.

\section{Conflict between Curative and Palliative Care}

The choice healthcare providers' face between providing curative and palliative care in healthcare settings is a prime example of goal conflict, with consequential implications for the health and well-being of others. Palliative care is treatment designed to manage pain and symptoms and provide psychosocial support in advanced illness or illness with high symptom burden, and can be administered even in conjunction with curative care (CAPC, 2015). In part due to the shortage of palliative specialists (Dharmarajan, Wei, \& Vapiwala, 2015), palliative care requires participation from providers at all levels, including not only physicians, but also nurses, nursing aids, medical support team members, pharmacists, and paramedics (Ahsberg \& Carlsson, 2014; Barrett \& Connaire, 2016; Kent et al., 2016; Morrison, Wallenstein, Natale, Senzel, \& Huang, 2000; Wiese et al., 2009). Palliative care decision making among healthcare providers is an ideal context in which to examine robustness of social psychological theory, 
because it involves high-stakes decisions made in a uniquely emotionally-laden environment, as well as decisions made on behalf of others (Ferrer, Padgett, \& Ellis, 2016).

The perceived conflict between curative and palliative care is an important real-world context to study goal conflict when making decisions on behalf of others for several reasons. First, healthcare providers tend to assume that such goal conflict exists, and prioritize curative goals, which suggests that they may be less likely to provide palliative care and delay conversations about prognosis and palliative care options (Bakitas, Lyons, Hegel, \& Ahles, 2013; CAPC, 2011; Gawande, 2014; Kelley \& Morrison, 2015; Mollica et al., under review; Peppercorn et al., 2011). Unfortunately, many providers erroneously believe that palliative care is appropriate only at end-of-life, and that patients may lose hope if it is provided (Garrett et al., 2014; Schenker et al., 2014; CAPC, 2011); thus, perceived conflict among curative and palliative goals may reflect conflict experienced by the provider, as well as perceived conflict projected onto the patient. Second, no research has experimentally investigated the effect of curativepalliative conflict on healthcare providers' decisions about whether to provide or refer patients to palliative care.

Finally, despite empirically supported benefits and expert recommendations, palliative care is underutilized in the U.S. (Cohen et al., 2008; Ferrell \& Grant, 2014; Ferrell, Temel, Temin, \& Smith, 2016; Ferrell et al., 2016; Ferris et al., 2009; Gidwandi et al., 2016; Graham, 2014). For example, cancer patients often receive chemotherapy within days of death without accompanying palliative care (Peppercorn et al., 2011), and dementia patients often undergo intensive and ineffective medical interventions in the last months of life (Mitchell et al., 2009). Palliative care has been shown to reduce symptom burden, lessen depression and anxiety, and improve physical and functional well-being among patients (Bakitas et al., 2015; El-Jawahri, 
Greer, \& Temel, 2011; Kavalieratos et al., 2016; Temel et al., 2010; Wright, Zhang, \& Ray, 2008) and their caregivers (Dionne-Odom et al., 2015; El-Jawahri et al., 2011; Wright et al., 2008). Palliative care also reduces pursuit of aggressive treatment options that are unlikely to be effective (Adelson et al., 2017; Temel et al., 2010). Accordingly, although most adults in the U.S. have limited knowledge of palliative care, the majority would want it for themselves or family members once they read the definition (CAPC, 2011). Critically, contrary to the perception that palliative care conflicts with curative care, palliative care does not hasten death and may even extend life for patients with terminal cancer (Bakitas et al., 2015; El-Jawahri et al., 2011; Temel et al., 2010; although see Kavalieratos et al., 2016), and pain management strategies (including opioid analgesics) do not interfere with curative care or lead to drug abuse or mortality (Novak, Nemeth, \& Lawson, 2004).

Thus, the decision whether to provide palliative care is an ideal context in which to study how goal conflict is negotiated and resolved in high stakes decision-making on behalf of others. Provision of palliative care involves serious consequences for the recipient of care: it can influence their pain, comfort, and longevity. The decision is commonly made by a variety of healthcare providers on behalf of patients. In addition, healthcare providers perceive a conflict between palliative care and curative care, even though the reality is that no such conflict exists. Therefore, we can take advantage of this pre-existing decision domain in order to manipulate perceptions of goal conflict that have the potential to serve as useful interventions that could serve as ways to improve treatment.

\section{Making Decisions for Other People}

The preceding sections reviewed evidence and theory in support of the notion that goal conflict influences individual decision-making when making choices for oneself. However, if 
such research is to be applied to cases in which people make important decisions for others, then it is important to consider the current understanding of how making decisions for other people may differ from making decisions for oneself. As stated, people often make decisions on behalf of others. Moreover, one person can set goals for another, and can engage in decisions and actions that help close others to achieve those goals (Fitzsimons et al., 2015; Fishbach \& Tu, 2016; Orehek \& Forest, 2016).

The available research suggests that people making decisions for others focus more on potential gains (Polman, 2012a; 2012b), are more indulgent (Laran, 2010), perceive fewer tradeoffs (Kray, 2000), and engage in more pre-decisional motivated reasoning (Polman, 2010), than when making decisions for themselves. When making a decision for someone else, people make more creative (Polman \& Emich, 2011) and idealistic (Laran, 2010; Lu, Xie, \& Xu, 2013) choices than when making a decision for themselves. Thus, although preliminary, it seems that people making decisions for others may be less constrained than when making decisions for themselves. Consistent with this assertion, at least one study suggested that people were more likely to decide in favor of chemotherapy when making the decision for someone else (either from the role as parent or role as healthcare provider) than when deciding for oneself (ZikmundFisher et al., 2006). However, other research suggests that both surrogates and providers have difficulty predicting others' preferences, often project their preferences onto patients, and use their own values and preferences when making decisions for others (e.g., Ditto et al., 2001; Fagerlin, Ditto, Danks, \& Houts, 2001; Norris et al., 2005; O’Donnell et al., 2003; Schneiderman et al., 1997). As such, even with the potential for less constraint on decisions for others, it seems that one's own individual decision-making tendencies are a strong influence on decisions for others. Importantly, each of these studies stopped short of specifically investigating how goal 
conflict would influence decision making when deciding for others.

Based on this existing evidence, it seems that, if anything, people who are making decisions for others may be more likely to choose in favor of the higher priority goal when faced with goal conflict. By focusing on potential gains (Polman, 2012a; b), making more indulgent choices (Laran, 2010), being idealistic (Laran, 2010; Lu et al., 2013), and being creative (Polman \& Emich, 2011), it seems that when making decisions for other people are inhibiting constraints imposed by conflicting goals. In addition, they seem to engage in motivated distortion (Polmam, 2010) in order to justify their actions by perceiving fewer tradeoffs (Kray, 2000) as they engage in such indulgent choices. Indeed, when faced with a chance to provide painful curative treatment (chemotherapy), people were more likely to adopt this option when they were making the decision for others than for themselves (Zikmund-Fisher et al., 2006). This suggestion is further supported by research which has found that a choice that is in conflict with alternatives can be appealing because it is presumed to be especially instrumental in attaining the prioritized goal (Kruglanski, Chernikova, Babush, Dugas, \& Schumpe, 2015; Kruglanski et al., 2014;

Orehek, Mauro, Kruglanski, \& van der Bles, 2012; Zhang, Fishbach, \& Kruglanski, 2007). Therefore, we predicted that healthcare providers would find palliative care less important and would be less willing to provide it when they perceived curative and palliative care to be in conflict. The present research is the first to experimentally manipulate perceptions of goal conflict between curative and palliative care. Importantly, although healthcare providers often assume curative and palliative care are in conflict, patient outcomes suggest that curative and palliative care are best presented as complementary treatments. Thus, this is a decision domain in which deciding for others in the face of goal conflict has important real-world consequences.

\section{Self-Affirmation}


In addition to a goal conflict manipulation, we also examined a brief social psychological induction with the potential to mitigate the consequences of goal conflict. Self-affirmation is a process by which individuals reflect on strengths or values (Cohen \& Sherman, 2014; Steele, 1988), which attenuates psychological dissonance associated with holding conflicting beliefs (Aronson, Cohen, \& Nail, 1999; Heine \& Lehman, 1997). Self-affirmation can also mitigate reactions to identity threat by bolstering a more global sense of self-competence (Cohen, Garcia, Apfel, \& Master, 2006; Cohen, Garcia, Purdie-Vaughns, Apfel, \& Brzustoski, 2009; Cohen \& Sherman, 2014; Hall, Zhao, \& Shafir, 2014; Havranek et al., 2012; Martens, Johns, Greenberg, \& Schimel, 2006). Healthcare providers have reported that medical competence imbues a sense of identity, and that being confronted with a medical problem they cannot solve is a threat to this identity (e.g., Gawande, 2014). Medical identity may be threatened by palliative care goals, because palliation may be seen as giving up (CAPC, 2011), and providers are less likely to initiate palliative care discussions when curative options are still available (Keating et al, 2010). Moreover, providers report that they find it difficult to deliver bad news, including prognostic information and palliative options (Fallowfield \& Jenkins, 2004; Mitchell, 2007).

Another reason self-affirmation may be particularly promising for attenuating goal conflict and its consequences in the domain of palliative care decision making is that selfaffirmation can facilitate other-directed positive emotions (Armitage \& Rowe, 2011; Crocker, Niiya, \& Mischkowski, 2008; Exline \& Zell, 2009), which may increase empathy with patients, thereby making their quality of life more salient and important to providers. Providers' inability to fully empathize with patients has been implicated in failure to adequately provide or refer to palliative care (Ferrer et al., 2016; Loewenstein, 2005). For example, physicians report that they themselves would not want to pursue aggressive curative measures with little chance for success 
(Garrett et al., 2014), but these preferences do not extend to their practices with patients. Closing this interpersonal hot-cold empathy gap could facilitate some perspective-taking and allow physicians to better calibrate preferences, shifting to palliative goals for care when necessary (see Kim et al., 2004). Thus, self-affirmation may increase the importance of palliative goals, regardless of whether they are in conflict with curative goals.

These two research streams seem to suggest that self-affirmation may reduce perceived curative-palliative goal conflict and increase importance of and willingness to administer palliative care. However, to the extent that self-affirmation interventions strengthen pre-existing values (Cohen et al., 2009; Yeager \& Walton, 2011), and healthcare providers currently value curative care and under-utilize palliative care, then it may instead increase perceived curativepalliative goal conflict and may decrease willingness to administer palliative care.

\section{The Present Research}

In the present study, we examined the consequences of goal conflict and self-affirmation in a large, nationally representative sample of U.S. healthcare providers, who made treatment decisions on behalf of others. We examined a range of providers, given that palliative care requires participation from providers at all levels, including not only physicians, but also nurses, nursing aids, medical support team members, pharmacists, and paramedics (Ahsberg \& Carlsson, 2014; Barrett \& Connaire, 2016; Kent et al., 2016; Morrison, Wallenstein, Natale, Senzel, \& Huang, 2000; Wiese et al., 2009). This underscores the need to understand malleable psychological factors that contribute to suboptimal palliative care provision/referral in a range of providers (Ferrer et al., 2016; Halpern, 2015; Padgett \& Ferrer, 2015). Providers were randomly assigned to self-affirm (vs. a neutral control condition), and to read a short message about the conflict (vs. complementarity) between curative and palliative goals for care. We then assessed 
importance of palliative care goals and willingness to provide palliative care in a specific scenario.

Due to prioritization of curative goals among healthcare providers, we predicted that goal conflict would lead providers to disengage from palliative goals, resulting in less willingness to provide or refer to palliative care (Hypothesis 1), consistent with evidence regarding how goal conflict influences choices among participants making decisions for themselves. We also predicted that self-affirmation would attenuate the consequences of goal conflict, increasing importance of palliative care goals and willingness to provide or refer to palliative care (Hypothesis 2). Finally, we predicted that goal conflict and self-affirmation would interact, such that self-affirmation would attenuate effects of goal conflict on importance and willingness (Hypothesis 3). The study methodology and hypotheses were pre-registered, and data are publicly available.

\section{Method}

The study proposal was selected for fielding by Time-Sharing Experiments in the Social Sciences (TESS; tessexperiments.org), a National Science Foundation funded initiative through which project proposals are vetted through a peer-review process and selected for funding based on whether the project is deemed meritorious by reviewers and the TESS Principal Investigators. Proposals (including hypotheses) are archived on the TESS website (in a process akin to preregistration), and data are made publicly available one year after a study is conducted (http://www.tessexperiments.org/previousstudies.html). The study was approved by the University of Pittsburgh Institutional Review Board. All measures, manipulations, and participant exclusions relevant to the study are reported.

\section{Participants}


Participants were a nationally representative U.S. sample of 502 healthcare providers recruited through a standing internet panel of non-institutionalized U.S. adults recruited using statistically valid sampling techniques, GfK Knowledge Panel (www.gfk.com). A priori power calculations determined that a sample size of 501 was yielded $95 \%$ power to detect differences in a $2 \times 2$ (goal conflict/ complementarity X self-affirmation/ no affirmation) factorial design, assuming a small-to-medium effect size. Sensitivity analyses indicated that a sample size of 502 can detect a small effect $(>f=0.16)$. Because the study was facilitated by TESS, sample size was determined based on the length of the survey, where longer surveys enroll fewer participants (see http://www.tessexperiments.org/limits.html). The specific inclusion criteria further reduced the allowable sample size. Therefore, to maximize power, we limited the length of the survey to accommodate $>501$ participants.

Table 1 contains demographic characteristics of the sample, which were derived from variables collected and provided by GfK (rather than reported as part of participation in the study). Although the sample was relatively highly educated and female because of the occupation targeted in the sampling strategy (i.e., healthcare providers), the sample was racially and ethnically diverse. Moreover, study weights were generated to render the sample representative of U.S. healthcare providers (see Analysis section). Providers included were in one of four categories: medical doctors ( $n=47$; e.g., physicians, surgeons); other healthcare practitioners ( $n=255$; e.g., nurses, pharmacists); healthcare support ( $n=116$; e.g., nursing aids, nursing assistants); and technologists/technicians ( $n=85$; e.g., paramedics, lab technicians). Because participants in the last occupational category may not necessarily interact with patients and thus may not have an opportunity to support referrals for palliative care, we conducted ancillary analyses excluding this occupational category. 


\section{Procedure and Materials}

The experiment was presented as two ostensibly separate studies. First, in the "values study," providers were randomly assigned to a self-affirmation condition (choosing their most important value from a list and writing for $\sim 8$ minutes about why the value is important to them and how they uphold it in daily life; see McQueen \& Klein, 2006) or a no-affirmation condition (writing about their daily routine; Burson, Crocker, \& Mischkowski, 2012).

Then, providers were transitioned to the "goals for care" study, in which they were randomly assigned to receive one of two messages about curative and palliative care. Both messages began by stating: "Palliative care is defined as an approach designed to treat pain, manage physical symptoms/treatment side effects, and provide psychosocial support that improves the quality of life of patients and their families." The goal conflict message went on to read:

However, barriers to integrating palliative care are many, and include important conflicts between integrating palliative and curative treatment, including medical training that prioritizes curative care no matter the cost, an incentive and payment structure weighted toward curative treatment, and a medical system that constrains time medical personnel with patients.

The goal complementarity message went on to read:

A growing number of medical organizations endorse the integration of palliative care into the entire care continuum of patients with advanced disease or significant symptom burden. Although there are barriers to optimal integration of palliative care, a mounting body of evidence shows that it can actually contribute to the benefits of curative care. For example, several recent studies have demonstrated that early palliative care can extend 
length of life among terminal cancer patients.

Participants then answered a questionnaire that included items assessing goal conflict, the interpersonal empathy gap, importance of palliative and curative goals, and willingness to provide or refer to palliative and curative care. The order of item presentation was randomized, such that half of the participants received the willingness item at the beginning of the survey, and half of the participants received this item at the end of the survey.

We developed items to assess curative and palliative goal conflict and goal complementarity, adapting a previously developed goal conflict matrix (Emmons \& King, 1988) and a pictorial item assessing overlap between the self and others (Aron \& Fraley, 1999). Items were assigned to each scale using exploratory factor analysis. We initially conducted an exploratory factor analysis (EFA) with no rotation, extracting factors based on an eigenvalue of greater than 1 with no set number of factors, which returned two distinct factors. Following the recommendations of Fabringer and colleagues (1999), we then conducted a second EFA with an oblique rotation (promax), specifying two factors, which indicated a low correlation among the factors ( $\mathrm{r}=-0.21$, below the recommended 0.32 threshhold for oblique rotation). As such, the final EFA involved an orthogonal rotation (varimax) to maximize the factor loadings, and extracted factors based on eigenvalue $>1$ with no set number of factors. All factor analyses specified principle components extraction. We used a cut-off value of 0.40 for factor loadings (all primary loadings > 0.42). One variable had a high cross-loading ("Palliative and curative care are in conflict with one another", primary loading $=0.65$, cross-loading $=-0.44)$, but because the primary loading was substantially higher than the cross-loading, it was assigned to the scale on which it had a higher loading (goal conflict). No other items had high cross loadings (all > $0.08)$. 
The Goal Conflict Scale $(\alpha=.73)$ comprised three items (1 strongly disagree -5

strongly agree): "Curative care is detrimental to palliative care;" "Palliative care is detrimental to curative care"" and "Palliative care and curative care are in conflict with one another." The Goal Complementarity Scale $(\alpha=.68)$ comprised five items. Two were on a scale from 1 very harmful to 5 very helpful: "Does being successful achieving palliative goals for a patient have a helpful, harmful, or no effect at all on achieving curative goals for a patient?" and "Does being successful achieving curative goals for a patient have a helpful, harmful, or no effect at all on achieving palliative goals for a patient?" The other two were on a scale from 1 strongly disagree to 5 strongly agree: "Curative care is helpful to palliative care" and "Palliative care is helpful to curative care." The fifth asked participants to select the image that best represents the relationship between curative and palliative care, presenting overlapping circles ranging from not overlapping to strongly overlapping. Because the latter item was on an 8-point scale, the goal complementarity items were standardized prior to score computation. Of note, the goal conflict and goal complementarity scales were only modestly correlated, $r=-0.29, p<.001, n=502$.

Importance of palliative goals was assessed with one item: "Palliative care is a strong priority" (1 strongly disagree - 7 strongly agree). Importance of curative care goals was assessed with one item: "Curative care is a strong priority" (1 strongly disagree - 7 strongly agree).

Palliative care willingness was assessed with one item: "If a patient requested a palliative procedure or treatment instead of a curative treatment, I would be willing to fulfill that request (if possible) or support them in making the request of other medical personnel” (1 strongly disagree - 7 strongly agree). Curative care willingness was also assessed with a single item: "If a patient requested a curative procedure or treatment that I did not believe would work, 
I would be willing to fulfill that request (if possible) or support them in making the request of other medical personnel" (1 strongly disagree - 7 strongly agree). These items were designed to assess willingness to provide these types of care under specific circumstances, patterned after behavioral willingness items shown to be more predictive of behavior than intentions items (Gibbons, Gerrard, Oulette, \& Burzette, 1998). The items included palliative and curative care situations in which providers may have some reluctance to provide care, to avoid ceiling effects. Participants were randomly assigned to have these items presented first or last in the outcome questionnaire, and they were counterbalanced.

Positive other-directed emotion was assessed with two items: "How much do you currently feel nurturing?" and "How much do you currently feel compassionate?" (1 not at all 7 extremely). Threat to medical identity was assessed with a single item: "I feel less competent when I am faced with a patient who has a medical problem that I cannot solve" (1 strongly disagree - 7 strongly agree). Empathy gap was operationalized as the difference between two counterbalanced items: "The patients I see who are dealing with disease or illness feel it is important to seek:" and "If you were a patient dealing with disease or illness, you would feel it is important to seek:" (1 curative care - 7 palliative care).

\section{Data Analytic Strategy}

In three separate $2 \times 2$ analyses of covariance (ANCOVA) performed in SAS 9.3, we examined whether the goal conflict condition (goal conflict vs. goal complementarity) increased 1) perceived goal conflict, and 2) decreased importance of palliative goals and 3) willingness to provide or support requests for palliative care (and whether the self-affirmation condition decreased these, as well as whether there was an interaction). In two separate regression analyses, using indirect modeling procedures available to evaluate mediation in Mplus 5, we 
examined whether: 1) Goal conflict condition had a causal indirect path to importance of palliative goals, mediated by perceived goal conflict; and 2) Goal conflict condition had a causal indirect path to willingness to provide or support requests for palliative care, mediated by perceived goal conflict.

We also conducted additional analyses not included in our pre-registered hypothesis and analysis plan. First, because we initially anticipated that perceived goal conflict and perceived goal complementarity would load onto the same factor (with perceived complementarity reversecoded), we did not include hypotheses about perceived complementarity as a mediator. However, as two factors emerged from this scale, we conducted analyses that included both perceived conflict and perceived complementarity as mediators, evaluating indirect effects via each individually and total indirect effects. Second, in response to a reviewer comment, we also examined whether the goal conflict condition influenced providers' ratings of patient preferences for curative versus palliative care.

We report analyses adjusted for age, gender, education (college vs. no college), race/ ethnicity (white vs. non-white), and occupation (physician vs. other healthcare provider), as well as unadjusted estimates. Order of the willingness item presentation did not influence responses, and was thus not included as a covariate. All analyses were weighted with post-stratification weights generated by GfK to attenuate biased statistical estimates that could be derived from the complex sampling design, in the context of the current experimental design (i.e., due to nonresponse and potential under-coverage within each experimental cell, as well as recruitment using different probability-based sampling methods as the panel composition changes over time) and render estimates nationally representative. Weights ensure that each experimental cell is representative of the population of interest (i.e., U.S. healthcare providers) and that analyses 
accurately reflect the demographic makeup of this population. Because Mplus will not calculate bias-corrected confidence intervals for weighted analyses, we used Monte Carlo simulation to estimate these paths (Selig \& Preacher, 2008), inputting weighted estimates of unstandardized paths and sampling variance, and specifying 95\% confidence intervals and 20,000 repetitions.

We also conducted ancillary analyses, repeating analytic procedures described above but excluding healthcare technologists and technicians from the sample. These individuals were included in the study because they may have a role informing decision making due to emergent crises or worsening symptoms associated with advanced disease. However, we acknowledge that many individuals in this occupational category may not interact with patients, and as such it is important that the pattern and reliability of analyses are reproduced when these individuals are excluded.

\section{Results}

Means, standard deviations, and ranges for all study variables are found in Table 2.

\section{Goal Conflict Induction}

Goal conflict and complementarity. As expected, participants in the goal conflict (vs. goal complementarity) condition reported increased perceived goal conflict, adjusted: $F(1,499)$ $=14.95, p<.001, d=0.35$, unadjusted: $F(1,499)=12.70, p=.004, d=0.32$, and decreased perceived goal complementarity, adjusted: $F(1,499)=5.66, p=.018, d=-0.21$, unadjusted: $F(1$, 499) $=5.77, p<.017, d=-0.21$.

Palliative goal importance. Participants in the goal conflict (vs. complementarity) condition also reported decreased importance of palliative care, adjusted: $F(1,499)=5.10, p=$ $.024, d=-0.20$, unadjusted: $F(1,499)=5.23, p=.023, d=-0.20$. There was also a causal indirect link between goal conflict (vs. complementarity) condition and importance of palliative 
goal importance, mediated by perceived goal conflict (adjusted: $B=-0.084,95 \% C I=-0.165$, 0.025, unadjusted: $B=-0.084,95 \% C I=-0.163,-0.025$; see Table 3 ), such that goal conflict (vs. complementarity) condition increased perceived goal conflict (adjusted: $B=0.28,95 \% C I=0.15$, $0.41, p<.001$, unadjusted: $B=0.27,95 \% C I=0.13,0.41, p=.001)$ and perceived goal conflict was negatively associated with importance of palliative goals (adjusted: $B=-0.30,95 \% C I=-$ $0.45,-0.16, p=.001$, unadjusted: $B=-0.31,95 \% C I=-0.45,-0.17, p<.001)$.

Curative goal importance. Goal conflict (vs. complementarity) condition also decreased the importance of curative care, adjusted: $F(1,499)=5.32, p=.022, d=-0.21$; unadjusted: $F(1$, $499)=4.92, p=.027, d=-0.20$. There was also a causal indirect link between goal conflict (vs. complementarity) condition and importance of curative goal importance, mediated by perceived goal conflict (adjusted: $B=-0.076,95 \% C I=-0.143,-0.024$, unadjusted: $B=-0.086,95 \% C I=-$ 0.158, -0.030; see Table 3), such that goal conflict (vs. complementarity) condition increased perceived goal conflict (adjusted: $B=0.28,95 \% C I=0.15,0.41, p<.001$, unadjusted: $B=0.27$, $95 \% C I=0.14,0.41, p=.001)$ and perceived goal conflict was negatively associated with importance of palliative goals (adjusted: $B=-0.27,95 \% C I=-0.39,-0.14, p<.001$, unadjusted: $B$ $=-0.32,95 \% C I=-0.43,-0.20, p<.001)$.

Palliative willingness. Although there was no direct effect of goal conflict condition on palliative care willingness, adjusted: $F(1,499)=1.38, p=.240, d=-0.11$, unadjusted: $F(1,499)$ $=1.33, p=.250, d=-0.10$, there was an indirect causal path from goal conflict (vs. complementarity) condition to palliative care willingness, mediated by perceived goal conflict (adjusted: $B=-0.072,95 \% C I=-0.143,-0.019$, unadjusted: $B=-0.083,95 \% C I=-0.161,-0.025$; see Table 3), such that goal conflict (vs. complementarity) condition increased perceived goal conflict (adjusted: $B=0.28,95 \% C I=0.15,0.41, p<.001$, unadjusted: $B=0.27,95 \% C I=0.13$, 
$0.41, p=.002$ ) and perceived goal conflict was negatively associated with palliative care willingness (adjusted: $B=-0.26,95 \% C I=-0.39,-0.12, p=.002$, unadjusted: $B=-0.31,95 \% C I=$ $-0.45,-0.17, p<.001)$

Curative willingness. There was no effect of goal conflict condition on willingness to provide curative care, adjusted: $F(1,499)=0.22, p=.643, d=-0.04$, unadjusted: $F(1,499)=$ $0.31, p=.575, d=-0.05$. Because perceived goal conflict was unassociated with willingness to provide curative care $(r=-0.05, p=.247, n=501)$, we did not examine whether there was an indirect effect of goal conflict condition on curative care willingness via perceived goal conflict. Although perceived goal complementarity was associated with willingness to provide curative care $(r=0.12, p=.006, n=501)$, there was no indirect effect of goal conflict condition on willingness to provide curative care via perceived goal complementarity adjusted: $(B=-0.040$, $95 \% C I=-0.104,0.002$, unadjusted: $B=-0.038,95 \% C I=-0.102,0.002$; see Table 3$)$.

Perceived patient preferences for curative vs. palliative care. There was no effect of goal conflict condition on perceptions of patients' preferences for curative vs. palliative care, adjusted: $F(1,499)=0.20, p=.651, d=0.04$, unadjusted: $F(1,499)=0.36, p=.647, d=0.05$

\section{Self-Affirmation}

Self-affirmation did not influence perceived goal conflict, adjusted: $F(1,499)=2.70, p=$ $.101, d=0.15$, unadjusted: $F(1,499)=2.61, p=.107, d=0.14$, or complementarity, $F(1,499)=$ $0.05, p=.821, d=0.02$, unadjusted: $F(1,499)=0.05, p=.816, d=0.02$, nor did it influence the importance of palliative care goals, adjusted: $F(1,499)=1.08, p=.300, d=-0.09$, unadjusted: $F(1,499)=1.06, p=.304, d=-0.09$. However, self-affirmation significantly reduced palliative care willingness, adjusted: $F(1,499)=4.37, p=.037, d=-0.19$, unadjusted: $F(1,499)=4.37, p$ $=.037, d=0.14$,. Self-affirmation had no effect on the interpersonal empathy gap, adjusted: $F(1$, 
499) $=0.48, p=.490, d=-0.06$, unadjusted: $F(1,499)=0.47, p=.448, d=-0.06$, other-directed positive emotions, adjusted: $F(1,499)=0.93, p=.336, d=-0.09$, unadjusted: $F(1,499)=0.83, p$ $=.364, d=-0.08$, or medical identity threat, adjusted: $F(1,499)=0.61, p=.435, d=-0.07$, unadjusted: $F(1,499)=0.61, p=.435, d=-0.07$,. There were no significant interactions between goal conflict condition and self-affirmation on any outcome variables.

\section{Ancillary Sensitivity Analyses}

Ancillary analyses excluding healthcare technologists/technicians revealed patterns of significant effects consistent with those described above. Specifically, goal conflict (vs. complementarity) condition increased perceived goal conflict, adjusted: $F(1,410)=19.33, p<$ $.001, d=0.44$, unadjusted: $F(1,410)=14.83, p<.001, d=0.38$, and the effect on perceived goal complementarity approached significance in the expected direction, adjusted: $F(1,410)=2.95, p$ $=.087, d=-0.17$. unadjusted: $F(1,410)=2.94, p=.087, d=-0.17$.

Goal conflict (vs. complementarity) condition reduced the importance of palliative goals among this sub-sample, adjusted: $F(1,410)=4.40, p=.037, d=-0.21$, unadjusted: $F(1,410)=$ $4.31, p=.038, d=-0.21)$. The indirect effect of goal conflict condition on importance of perceived goal conflict via perceived goal conflict and perceived goal complementarity (individually) had a confidence interval including 0 in this sample, but the total indirect effect of goal conflict condition via these two mediators remained significant (Table 3).

Goal conflict (vs. complementarity) condition also reduced the importance of curative goals among this sub-sample, adjusted: $F(1,410)=5.25, p=.023, d=-0.23$, unadjusted: $F(1$, $410)=4.35, p=.038, d=-0.21$. The indirect effect of goal conflict condition via perceived goal conflict, and the total indirect effect of goal conflict condition via perceived goal conflict and complementarity together, remained significant for importance of curative goals in this sub- 
sample; however, the indirect effect of goal conflict condition on importance of curative goals via perceived goal complementarity had a confidence interval overlapping 0 (Table 3).

When technologists/technicians were removed from analyses, there was a direct effect of goal conflict (vs. complementarity) condition on palliative care willingness, adjusted: $F(1,410)$ $=4.45, p=.035, d=-0.21$, although when unadjusted the effect was no longer significant: $F(1$, $410)=3.77, p=.053, d=0.19$. The indirect effect of goal conflict condition via perceived goal conflict, and the total indirect effect of goal conflict condition via perceived goal conflict and complementarity together, remained significant for palliative care willingness in this sub-sample; however, the indirect effect of goal conflict condition on palliative care willingness via perceived goal complementarity had a confidence interval overlapping 0 (Table 3).

There was also a significant effect of self-affirmation, adjusted: $F(1,410)=4.04, p=$ $.045, d=-0.20$, which was again not significant when unadjusted, $F(1,410)=2.45, p=.064, d=$ -0.18 .

\section{Discussion}

This experimental study investigated the consequences of goal conflict when making decisions for others in a high-stakes decision-making context in which healthcare providers make decisions for patients (see Ferrer et al., 2016). Among a nationally representative sample of U.S. healthcare providers, we found that a message presenting curative and palliative goals as in conflict (as opposed to as complementary to one another) increased perceived goal conflict and decreased goal complementarity, as well as decreased the importance of both curative and palliative goals for care. To our knowledge, this is the first study to examine the consequences of goal conflict among people making decisions on behalf of others, among experts, and in a highstakes decision making context, as well as the first to point to goal conflict as a malleable barrier 
to optimal palliative care uptake.

We predicted that providers would resolve goal conflict for a decision made on behalf of others following strategies used for resolving goal conflict for an individual choice. Specifically, because providers often value curative goals over palliative goals (Bakitas et al., 2013; Gawande, 2014; Mollica et al., 2018), and project these preferences to their patients (Fagerlin et al., 2001; Norris et al., 2005; O’Donnell et al., 2003; Schneiderman et al., 1997), we expected that goal conflict (vs. complementarity) condition would attenuate the importance of palliative, but not curative, goals, consistent with the tendency for individuals to resolve conflict by pursuing the prioritized goal and disengaging from the less prioritized goal (Achtziger et al., 2008; Gollwitzer et al., 1990; Fishbach \& Zhang, 2008; Kruglanski et al., 2002; Köpetz et al., 2008; Shah \& Kruglanski, 2002). However, we found that goal conflict (vs. complementarity) condition reduced the importance of both types of goals. It is possible that the goal complementarity condition was driving this effect - that is, that providers' perceiving the two goals as complementary were able to value both concurrently. This explanation is consistent with research demonstrating individuals are better able to pursue concurrent goals when they can be pursued by a single means or when they are otherwise not conflicting (Chun, Kruglanski, SleethKeppler, \& Friedman, 2011; Kruglanski et al., 2013). However, mediation analyses indicate that both perceived goal conflict and perceived goal complementarity mediate effects on goal importance. This suggests that goal complementarity may not fully explain these findings. It is possible that individuals disengage from both goals when facing goal conflict on behalf of others, or that providers were responding to the goal conflict message by prioritizing a third, unmeasured goal. Future research should examine these possibilities.

Conversely, effects of goal conflict (vs. complementarity) on willingness to provide or 
refer to palliative care and willingness to provide aggressive curative care were largely consistent with the notion that individuals resolve goal conflict when making decisions for others by disengaging from the less important goal. Although the goal conflict induction did not have a direct influence on willingness to provide palliative care, there was an indirect effect of condition on willingness, mediated by perceived goal conflict (but not perceived complementarity). The absence of a direct effect does not preclude mediation (Hayes, 2009; Shrout \& Bolger, 2002), and indirect effects are often observed in absence of a detectable direct effect (O'Rourke \& MacKinnon, 2015). Indirect effects in absence of direct effects suggest either a direct effect too small to detect (Kenny \& Judd, 2014), given that a smaller $\mathrm{n}$ is necessary to detect an indirect effect compared to a direct effect, or can point to the presence of an unmeasured opposing force (Hayes, 2009; Shrout \& Bolger, 2002). These possibilities cannot be examined in the available data, and should be addressed in future research; however, the presence of an indirect effect, even without a direct effect, points to an influence of goal conflict on palliative care willingness. Conversely, the goal conflict induction did not influence willingness to provide curative care directly or indirectly. These two findings, taken together, are consistent with our hypotheses, but not with findings for goal importance. Future research should examine why goal importance did not translate neatly to willingness.

We found that the goal conflict condition did not influence providers' ratings of patients' prioritization of curative vs. palliative care. As such, although we do not have an analogous assessment of patients' goal importance, we can infer that the effect of the goal conflict induction is not influencing goal importance and behavioral willingness via changes to providers' understanding of patient goals. Rather, the goal conflict appears to be experienced and resolved based on the providers' goals, rather than the patients' goals. This finding is consistent with 
research on surrogate decision making suggesting that providers' behaviors are guided by their own values (and that they are often unable to accurately estimate their patients' values; e.g., Carmel et al., 1999; Downey et al., 2013; Druley et al., 2013; Fagerlin, Ditto, Danks, \& Houts, 2001; Figueroa et al., 2016; Gramelspacher et al., 1997; Norris et al., 2005; O’Donnell et al., 2003; Schneiderman et al., 1997).

Interestingly, self-affirmation reduced willingness to provide or support requests for palliative care, contrary to expectations that it might have a beneficial effect because of its effects on reducing cognitive dissonance (Aronson et al., 1999; Heine \& Lehman, 1997) increasing positive, other-directed affect (Armitage \& Rowe, 2011; Crocker et al., 2008; Exline \& Zell, 2009), and attenuating threat to medical identity (Cohen et al., 2006; 2009; Cohen \& Sherman, 2014; Hall et al., 2013; Hravanek et al., 2012; Martens et al., 2006). Although contrary to predictions, there is precedent for reversal of self-affirmation's effects under certain conditions (Briñol, Petty, Gallardo, \& DeMarree, 2007; Critcher, Dunning, \& Armor, 2010; Ferrer, Klein, \& Graff, 2017). Meta-analytic evidence suggests that self-affirmation is only beneficial to the extent that attention is paid to appropriate transitional timing and resources to support attitude and behavior change (Ferrer \& Cohen, under review), two factors that were not considered explicitly in this design. Moreover, self-affirmation can result in disengagement from difficult (and potentially less self-relevant or valued) goals (Vohs, Park, \& Schmeichel, 2013), as self-affirmation strengthens only behavioral pursuit of valued goals (Cohen et al., 2009; Yeager \& Walton, 2011). As stated, healthcare providers value curative over palliative care goals (Bakitas et al., 2013; Gawande, 2014).

This study has important implications for improving quality of life among individuals with disease and their caregivers. Increasing the importance of palliative goals for care across a 
spectrum of healthcare providers is important, particularly given recent efforts to integrate palliative care into primary and specialty care to combat shortages of specialized palliative care providers (Dharmarajan, Wei, \& Vapiwala, 2015; Kelley \& Morrison, 2015). This study demonstrates that conflict among curative and palliative goals reduces the importance of palliative (and curative) goals, and that presenting the two as complementary increases the importance of palliative (and curative) goals. The consequences of goal conflict among providers may be self-perpetuating, given that limited time in clinical visits (Nutting, 1994; Walter, Li, Dunsmuir, \& Westbrook, 2014) may contribute to perceived conflict between curative and palliative goals for care, and that goal conflict, in turn, can reduce perceptions of available time (Etkin, Evangelidis, \& Aaker, 2015).

As healthcare providers place higher value in palliative goals, the benefits of palliative care - including improved outcomes among both patients and caregivers (Bakitas et al., 2015; Dionne-Odom et al., 2015; El-Jawahri et al., 2011; Temel et al., 2010; Wright et al., 2008), decreased use of aggressive treatment options unlikely to be effective (Adelson et al., 2017; Temel et al., 2010), and decreased healthcare costs (Goldfield, Stevenson, Hamel, \& Mitchell, 2011; Obermeyer et al., 2014) - will be more fully realized. Our brief goal complementarity message facilitated positive attitudes towards palliative care, compared to the control condition, without decreasing - in fact, increasing - attitudes towards curative care. The efficacy of the message should be interpreted with caution, as the design was intended to uncover causal evidence for goal conflict's role in providers' palliative care importance and willingness, and thus did not include a true control condition. However, whether using this brief method or another method, social psychological interventions targeting goal conflict have the potential to improve palliative care uptake and quality of life outcomes. Indeed, conflict between curative 
and palliative goals may be particularly amenable to intervention, given evidence supporting the complementarity of these (i.e., trials showing that palliative care may extend life; Bakitas et al., 2015; Temel et al., 2010), and highlighting ways in which a single behavior can be used to achieve multiple goals can attenuate perceived goal conflict (Chun et al., 2011; Köpetz et al., 2011; Kruglanski et al., 2013; Orehek et al., 2012; Orehek \& Vazeou-Nieuwenhuis, 2013). However, our message was clear and straightforward, and it may be more difficult to facilitate perceptions of curative-palliative goal complementarity in complex real-world medical situations.

One limitation of this study is the use of single-item assessments of outcomes. However, single-item assessments of social-cognitive and decision-making constructs are common in large-scale survey research (e.g., Ferrer et al., 2011; Ferrer, Portnoy, \& Klein, 2013), and single items can have similar construct validity and test-retest reliability, compared to validated multiitem scales (Ferrer, Klein, Avishai-Yitshak, Jones, \& Sheeran, under review; Bergkvist \& Rossiter, 2007). Nonetheless, single items are less reliable than scales and as such may have attenuated some of the associations observed in the study (Luttrell, Petty, and Xu, 2017). Findings related to the willingness item - which assessed willingness to provide palliative care instead of curative care - are also limited by the sample, of whom some (unknown) proportion are less involved in treatment of patients with potentially terminal diagnoses and therefore answering this item hypothetically. This item was intentionally constructed to examine behavior under specific circumstances, as specific behavioral willingness items are more predictive of actual behavior than are vaguer intentions items (Gibbons, Gerrard, Ouelette, \& Burzette, 1998). However, importance of palliative care is relevant to the full sample regardless of their experience with terminal patients, given that palliative care is appropriate in medical care 
regardless of whether the patient has a terminal diagnosis. These limitations are offset by a number of strengths, including use of a large nationally representative sample of U.S. healthcare providers and use of an experimental design that allows for causal inferences.

\section{Conclusion}

To our knowledge, this study is the first to elucidate the dynamics of goal conflict among experts making decisions for others and for high-stakes decision making. This study also elucidates a malleable social psychological mechanism serving as a barrier to palliative care: goal conflict among healthcare providers. Because perceived goal conflict can be reduced or eliminated via low-burden interventions, this study points to a clear method to increase palliative care utilization, while concurrently increasing the importance of curative goals. Future studies should examine the dynamics of and consequences for goal conflict among other sets of experts, and for other high-stakes decision-making contexts. This study provides a model for leveraging ecologically valid, consequential health contexts for understanding the robustness of social psychological theory regarding goal conflict, as well as enriching health care by looking beyond disciplinary "silos" to better understand and solve clinical challenges. 
Table 1. Sociodemographic characteristics of sample

\begin{tabular}{|c|c|c|}
\hline \multirow[b]{2}{*}{ Age } & \multirow{2}{*}{\multicolumn{2}{|c|}{$\begin{array}{l}\begin{array}{l}\text { Weighted } \\
\text { Mean (SD) }\end{array} \\
4280(1304)\end{array}$}} \\
\hline & & $20-79$ \\
\hline & Number & $\begin{array}{r}\text { Weighted } \\
\text { Percent }\end{array}$ \\
\hline Female & 372 & $79.23 \%$ \\
\hline \multicolumn{3}{|l|}{ Occupation } \\
\hline Medical Doctor & 47 & $7.02 \%$ \\
\hline Other Healthcare Practitioner & 255 & $51.76 \%$ \\
\hline Healthcare Support & 116 & $24.46 \%$ \\
\hline Health Technologist or Technician & 85 & $16.75 \%$ \\
\hline \multicolumn{3}{|l|}{ Education } \\
\hline Less than high school & 9 & $2.27 \%$ \\
\hline High school & 58 & $14.48 \%$ \\
\hline Some college & 184 & $37.09 \%$ \\
\hline College & 252 & $46.16 \%$ \\
\hline \multicolumn{3}{|l|}{ Race/ Ethnicity } \\
\hline White, non-Hispanic/ Latino(a) & 353 & $63.97 \%$ \\
\hline Black, non-Hispanic/ Latino(a) & 52 & $14.88 \%$ \\
\hline Other, non-Hispanic/ Latino(a) & 23 & $8.95 \%$ \\
\hline 2+ Races, non-Hispanic/ Latino(a) & 54 & $1.58 \%$ \\
\hline Hispanic/ Latino(a) & 21 & $10.62 \%$ \\
\hline
\end{tabular}


Table 2. Weighted means, standard deviations, and ranges of study variables

\begin{tabular}{lrr}
\hline & $\begin{array}{r}\text { Weighted } \\
\text { Mean (SD) }\end{array}$ & Range \\
\cline { 2 - 3 } Goal Conflict & $2.50(0.87)$ & $1-7$ \\
Goal Fusion & $0.00(0.66)$ & $-2.60-1.58$ \\
Palliative Care Importance & $5.37(1.34)$ & $1-7$ \\
Curative Care Importance & $5.45(1.22)$ & $1-7$ \\
Palliative Care Willingness & $5.69(1.43)$ & $1-7$ \\
Curative Care Willingness & $5.19(1.54)$ & $1-7$ \\
Positive Other-directed Emotion & $5.52(1.14)$ & $1-7$ \\
Empathy Gap & $0.10(1.37)$ & $-6-6$ \\
Medical Identity Threat & $4.17(1.72)$ & $1-7$ \\
\hline
\end{tabular}


Table 3. Indirect effects of goal conflict condition on study outcomes, mediated by perceived goal conflict and complementarity.

\begin{tabular}{|c|c|c|c|c|c|c|c|c|c|c|c|}
\hline \multirow[b]{3}{*}{ Full sample } & \multicolumn{4}{|c|}{ Indirect via Perceived Goal Conflict } & \multicolumn{4}{|c|}{ Indirect via Perceived Goal Complementarity } & \multicolumn{3}{|c|}{ Total Indirect Effect } \\
\hline & \multicolumn{2}{|c|}{ Adjusted } & \multicolumn{2}{|c|}{ Unadjusted } & \multicolumn{2}{|c|}{ Adjusted } & \multicolumn{2}{|c|}{ Unadjusted } & \multicolumn{2}{|c|}{ Adjusted } & Unadjusted \\
\hline & $B$ & $95 \% C I$ & $B$ & $95 \% C I$ & $B$ & $95 \% C I$ & $B$ & $95 \% C I$ & $B$ & $95 \% C I$ & $B \quad 95 \% C I$ \\
\hline Palliative Impc & -0.048 & $.112,-0.001$ & .051 & $.116,-0.005$ & .081 & $-0.172,-0$ & 0.082 & 0 & .129 & $0.210,-0.048$ & $0.133-0.216,-0$ \\
\hline & .047 & $-0.104,-0.004$ & -0.062 & $-0.122,-0.018$ & .061 & -0.6 & & & & $74,-0.043$ & $-0.121-0.190,-0$ \\
\hline & -0.055 & $-0.113,-0.013$ & -0.068 & $-0.139,-0.015$ & & & & & -0.091 & $-0.148,-0.033$ & $-0.103-0.166,-0.041$ \\
\hline & 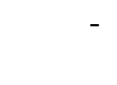 & & 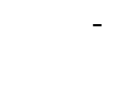 & & & & 38 & & & & $-\quad-$ \\
\hline tance & -0.063 & $-0.147,0.004$ & -0.055 & & -0.066 & & -0.066 & & -0.129 & $-0.223,-0.034$ & $-0.121-0.214,-0.029$ \\
\hline & .063 & $-0.139,-0.004$ & -0.079 & $-0.152,-0.6$ & -0.046 & & & & & $83,-0.036$ & $-0.124-0.200,-0.047$ \\
\hline & & $-0.115,0.017$ & -0.062 & $-0.141,-0.004$ & & & & & & $-0.132,-0.008$ & $-0.090-0.156,-0.025$ \\
\hline Curative Willingness & & - & - & & -0.009 & $-0.091,0.009$ & -0.031 & $-0.098,0.011$ & & & 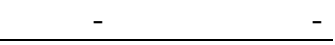 \\
\hline
\end{tabular}

Note: Italicized cells indicate a 95\% confidence interval that does not include 0 . 
Figure 1. Structural equation model

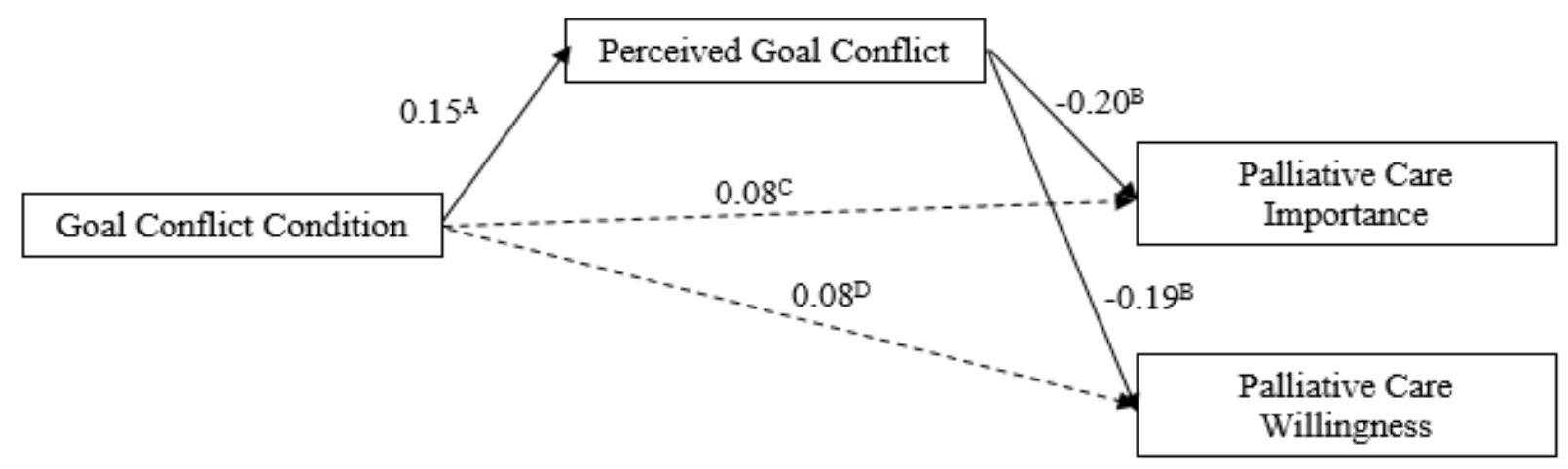

Note: Solid lines indicate direct effects; dotted lines indicate indirect effects. Analyses controlled for age, gender, education, and race/ethnicity, and incorporated post-stratification weights to attenuate sampling bias and render estimates nationally representative.

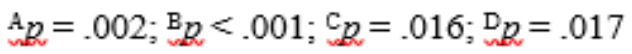




\section{Open Practices}

The study proposal was selected for fielding by Time-Sharing Experiments in the Social

Sciences (TESS; tessexperiments.org). The proposal and all hypotheses were peer-reviewed, and the study was conducted based on this pre-registered protocol. The study proposal and all study data are available at http://www.tessexperiments.org/data/ferrer825.html. The proposal, methods section, and data labels are sufficient to allow a third-party researcher to replicate all reported results. 


\section{References}

Achtziger, A., Gollwitzer, P. M., \& Sheeran, P. (2008). Implementation intentions and shielding goal striving from unwanted thoughts and feelings. Personality and Social Psychology Bulletin, 34, 381-393.

Adelson, K., Paris, J., Horton, J. R., Hernandez-Tellez, L., Ricks, D., Morrison, R. S., \& Smith, C. B. (2017). Standardized Criteria for Palliative Care Consultation on a Solid Tumor Oncology Service Reduces Downstream Health Care Use. Journal of Oncology Practice, JOP-2016.

Ahsberg, E., \& Carlsson, M. (2014). Practical care work and existential issues in palliative care: experiences of nursing assistants. International Journal of Older People Nursing, 9, 298-305.

Armitage, C. J., \& Rowe, R. (2011). Testing multiple means of self-affirmation. British Journal of Psychology, 102(3), 535-545.

Aron, A., \& Fraley, B. (1999). Relationship closeness as including other in the self: Cognitive underpinnings and measures. Social Cognition, 17, 140-160.

Aronson, J., Cohen, G. L., \& Nail, P. R. (1999). Self-affirmation theory: An update and appraisal. In E. Harmon-Jones \& J. Mills (Eds.). Cognitive dissonance theory: Revival with revisions and controversies (pp.127-147). Washington, DC: American Psychological Association.

Azoulay, E., Pochard, F., Kentish-Barnes, N., Chevret, S., Aboab, J., Adrie, C., Annane, D., Bleichner, G., Bollaert, P. E., Darmon, M., \& Fassier, T. (2005). Risk of post-traumatic stress symptoms in family members of intensive care unit patients. American Journal of Respiratory and Critical Care Medicine, 171, 987-994. 
Bakitas, M., Lyons, K. D., Hegel, M. T., \& Ahles, T. (2013). Oncologists' perspectives on concurrent palliative care in a National Cancer Institute-designated comprehensive cancer center. Palliative and Supportive Care, 11, 415-423.

Bakitas, M., Tosteson, T., Li, Z., Lyons, K. D., Hull, J. G., Li, Z., . . Ahles, T. (2015). Early versus delayed initiation of concurrent palliative oncology care: patient outcomes in the ENABLE III randomized controlled trial. Journal of Clinical Oncology, 33, 1438-1445.

Barrett, M., \& Connaire, K. (2016). A palliative care approach in heart failure: knowledge and attitudes of cardiac nurses. British Journal of Cardiac Nursing, 11, 136-142.

Bélanger, J. J., Kruglanski, A. W., Chen, X., \& Orehek, E. (2014). Bending perception to desire: Effects of task demands, motivation, and cognitive resources. Motivation and Emotion, 38, 802-814.

Bélanger, J. J., Kruglanski, A. W., Chen, X., Orehek, E., \& Johnson, D. J. (2015). When Mona Lisa smiled and love was in the air: On the cognitive energetics of motivated judgments. Social Cognition, 33, 104-119.

Briñol, P., Petty, R. E., Gallardo, I., \& DeMarree, K. G. (2007). The effect of self-affirmation in nonthreatening persuasion domains: Timing affects the process. Personality and Social Psychology Bulletin, 33, 1533-1546.

Burson, A., Crocker, J., \& Mischkowski, D. (2012). Two types of value-affirmation: Implications for self-control following social exclusion. Social Psychological and Personality Science, 3, 510-516.

CAPC. (2011). 2011 Public opinion research on palliative care: a report based on research by public opinion strategies. Retrieved from http://www.capc.org/tools-for-palliative-care- 
programs/marketing/public-opinion-research/2011-public-opinion-research-on-palliativecare.pdf

CAPC. (2015). Palliative Care Definition.

Chun, W. Y., Kruglanski, A. W., Sleeth-Keppler, D., \& Friedman, R. S. (2011). Multifinality in implicit choice. Journal of Personality and Social Psychology, 101, 1124-1137.

Cohen, E., Botti, M., Hanna, B., Leach, S., Boyd, S., \& Robbins, J. (2008). Pain beliefs and pain management of oncology patients. Cancer Nursing, 31, E1-E8.

Cohen, G. L., Garcia, J., Apfel, N., \& Master, A. (2006). Reducing the racial achievement gap: A social-psychological intervention. Science, 313, 1307-1310.

Cohen, G. L., Garcia, J., Purdie-Vaughns, V., Apfel, N., \& Brzustoski, P. (2009). Recursive processes in self-affirmation: Intervening to close the minority achievement gap. Science, 324, 400-403.

Cohen, G. L., \& Sherman, D. K. (2014). The psychology of change: Self-affirmation and social psychological intervention. Annual Review of Psychology, 65, 333-371.

Critcher, C. R., Dunning, D., \& Armor, D. A. (2010). When self-affirmations reduce defensiveness: Timing is key. Personality and Social Psychology Bulletin, 36, 947-959.

Crocker, J., Niiya, Y., \& Mischkowski, D. (2008). Why does writing about important values reduce defensiveness? Self-affirmation and the role of positive other-directed feelings. Psychological Science, 19, 740-747.

Dharmarajan, K. V., Wei, R., \& Vapiwala, N. (2015). Primary palliative care education in specialty oncology training: More work is needed. JAMA Oncology, 2, 858-859.

Dionne-Odom, J. N., Azuero, A., Lyons, K. D., Hull, J. G., Tosteson, T., Li, Z., \& Bakitas, M. (2015). Benefits of early versus delayed palliative care to informal family caregivers of 
patients with advanced cancer: Outcomes from the ENABLE III randomized controlled trial. Journal of Clinical Oncology, 33, 1446-1452.

El-Jawahri, A., Greer, J. A., \& Temel, J. S. (2011). Does palliative care improve outcomes for patients with incurable illness? A review of the evidence. Journal of Supportive Oncology, 9, 87-94.

El-Jawahri, A., LeBlanc, T., VanDusen, H., Traeger, L., Greer, J. A., Pirl, W. F., . . Temel, J. S. (2016). Effect of Inpatient Palliative Care on Quality of Life 2 Weeks After Hematopoietic Stem Cell Transplantation: A Randomized Clinical Trial. Journal of the American Medical Association, 316, 2094-2103.

Emmons, R. A., \& King, L. A. (1988). Conflict among personal strivings: immediate and longterm implications for psychological and physical well-being. Journal of personality and social psychology, 54, 1040-1048.

Etkin, J., Evangelidis, I., \& Aaker, J. (2015). Pressed for time? Goal conflict shapes how time is perceived, spent, and valued. Journal of Marketing Research, 52, 394-406.

Exline, J. J., \& Zell, A. L. (2009). Empathy, self-affirmation, and forgiveness: The moderating roles of gender and entitlement. Journal of Social and Clinical Psychology, 28, 1071-1099.

Fallowfield, L., \& Jenkins, V. (2004). Communicating sad, bad, and difficult news in medicine. The Lancet, 363, 312-319.

Ferrell, B., \& Grant, M. (2014). The future of palliative care. Seminars in Oncology Nursing, 30, 296-297.

Ferrell, B. R., Temel, J. S., Temin, S., \& Smith, T. J. (2016). Integration of Palliative Care Into Standard Oncology Care: ASCO Clinical Practice Guideline Update Summary. Journal of Oncology Practice, 13, 119-121. 
Ferrer, R. A., Klein, W. M., \& Graff, K. A. (2017). Self-affirmation increases defensiveness toward health risk information among those experiencing negative emotions: Results from two national samples. Health Psychology, 36, 380-391.

Ferrer, R. A., Padgett, L., \& Ellis, E. M. (2016). Extending emotion and decision-making beyond the laboratory: The promise of palliative care contexts. Emotion, 16, 581-586.

Ferris, F. D., Bruera, E., Cherny, N., Cummings, C., Currow, D., Dudgeon, D., ... Von Roenn, J. H. (2009). Palliative cancer care a decade later: accomplishments, the need, next stepsfrom the American Society of Clinical Oncology. Journal of Clinical Oncology, 27, 30523058.

Fishbach, A., \& Tu, Y. (2016). Pursuing Goals with Others. Social and Personality Psychology Compass, 10(5), 298-312.

Fishbach, A., \& Zhang, Y. (2008). Together or apart: when goals and temptations complement versus compete. Journal of Personality and Social Psychology, 94, 547-559.

Fitzsimons, G. M., Finkel, E. J., \& Vandellen, M. R. (2015). Transactive goal dynamics. Psychological Review, 122(4), 648-673.

Garrett, M., Chinn, G. M., Liu, P., Klabunde, C. N., \& Kahn, K. L. (2014). Physicians’ preferences for hospice if they were terminally ill and the timing of hospice discussions with their patients (research letter) JAMA: Journal of the American Medical Association, 174, 465-467.

Gawande, A. (2014). Being Mortal: medicine and what matters in the end. Doubleday Canada.

Gibbons, F. X., Gerrard, M., Ouelette, J. D., \& Burzette, R. (1998). Cognitive antecedents to adolescent health risk: Discriminating between behavioral intention and behavioral willingness. Psychology \& Health, 13, 319-339. 
Gidwandi, R., Joyce, N., Kinosian, B., Faricy-Anderson, K., Levy, C., Miller, S. C., . . Mor, V. (2016). Gap between Recommendations and Practice of Palliative Care and Hospice in Cancer Patients. Journal of Palliative Medicine, 19, 957-963.

Goldfield, K. S., Stevenson, D. G., Hamel, M. B., \& Mitchell, S. L. (2011). Medicare expenditures among nursing home residents with advanced dementia. Archives of Internal Medicine, 171, 824-830.

Gollwitzer, P. M., Heckhausen, H., \& Steller, B. (1990). Deliberative and implemental mindsets: Cognitive tuning toward congruous thoughts and information. Journal of Personality and Social Psychology, 59, 1119-1127.

Goschke, T., \& Dreisbach, G. (2008). Conflict-triggered goal shielding: Response conflicts attenuate background monitoring for prospective memory cues. Psychological Science, 19, 25-32.

Graham, J. (2014). IOM Report Calls for Transformation of End-of-Life Care. JAMA: Journal of the American Medical Association, 312, 1845-1847.

Hall, C. C., Zhao, J., \& Shafir, E. (2014). Self-affirmation among the poor: Cognitive and behavioral implications. Psychological Science, 25, 619-625.

Halpern, S. D. (2015). Towards evidence-based end-of-life care. N Engl J Med, 373, 2001-2003.

Havranek, E. P., Hanratty, R., Tate, C., Dickinson, L. M., Steiner, J. F., Cohen, G., \& Blair, I. A. (2012). The effect of values affirmation on race-discordant patient-provider communication. Archives of Internal Medicine, 172, 1662-1667.

Heine, S. J., \& Lehman, D. R. (1997). Culture, dissonance, and self-affirmation. Personality and Social Psychology Bulletin, 23, 389-400. 
Kavalieratos, D., Corbelli, J., Zhang, D., Dionne-Odom, J. N., Ernecoff, N. C., Hamner, J., . . . Schenker, Y. (2016). Association Between Palliative Care and Patient and Caregiver Outcomes: A Systematic Review and Meta-analysis. Journal of the American Medical Association, 316, 2104-2114.

Keating, N. L., Landrum, M. B., Rogers, S. O., Baum, S. K., Virnig, B. A., Huskamp, H. A., ... \& Kahn, K. L. (2010). Physician factors associated with discussions about end-of-life care. Cancer, 116(4), 998-1006.

Kelley, A. S., \& Morrison, R. S. (2015). Palliative care for the seriously ill. New England Journal of Medicine, 2015, 747-755.

Kent, E. E., Ferrer, R. A., Mollica, M., Huang, G., Falisi, A., \& Chou, W. Y. S. (2016). What does 'palliative care' mean? Diverse definitions from cancer care providers. ASCO Annual Meeting Proceedings, 30, 40.

Kim, S. S., Kaplowitz, S., \& Johnston, M. V. (2004). The effects of physician empathy on patient satisfaction and compliance. Evaluation \& the health professions, 27(3), 237-251.

Köpetz, C., Faber, T., Fishbach, A., \& Kruglanski, A. W. (2011). The multifinality constraints effect: how goal multiplicity narrows the means set to a focal end. Journal of Personality and Social Psychology, 100, 810-826.

Köpetz, C. E., Kruglanski, A. W., Chen, X., \& Orehek, E. (2008). Goal systemic effects in the context of choice and social judgment. Personality and Social Psychology Compass, 2, 20712089.

Köpetz, C., \& Orehek, E. (2015). When the end justifies the means: Self-defeating behaviors as "rational" and "successful" self-regulation. Current Directions in Psychological Science, 24, 386-391. 
Kray, L. J. (2000). Contingent weighting in self-other decision making. Organizational Behavior and Human Decision Processes, 83, 82-106.

Kruglanski, A. W., Chernikova, M., Babush, M., Dugas, M., \& Schumpe, B. (2015). The architecture of goal systems: Multifinality, equifinality, and counterfinality in means-end relations. Advances in Motivation Science, 2, 69-98.

Kruglanski, A. W., Gelfand, M. J., Bélanger, J. J., Sheveland, A., Hetiarachchi, M., \& Gunaratna, R. (2014). The psychology of radicalization and deradicalization: How significance quest impacts violent extremism. Political Psychology, 35, 69-93.

Kruglanski, A. W., Köpetz, C., Bélanger, J. J., Chun, W. Y., Orehek, E., \& Fishbach, A. (2013). Features of multifinality. Personality and Social Psychology Review, 17, 22-39.

Kruglanski, A. W., Shah, J. Y., Fishbach, A., Friedman, R., Chun, W. Y., \& Sleeth-Keppler, D. (2002). A theory of goal systems. Advances in Experimental Social Psychology, 34, 331-378.

Laran, J. (2010). Goal management in sequential choices: Consumer choices for others are more indulgent than personal choices. Journal of Consumer Research, 37, 304-314.

Loewenstein, G. (2005). Hot-cold empathy gaps and medical decision making. Health Psychology, 24(4S), S49-S56.

Lu, J., Liu, Z., \& Fang, Z. (2016). Hedonic products for you, utilitarian products for me. Judgment and Decision Making, 11, 332-341.

Martens, A., Johns, M., Greenberg, J., \& Schimel, J. (2006). Combating stereotype threat: The effect of self-affirmation on women's intellectual performance. Journal of Experimental Social Psychology, 42, 236-243.

McQueen, A., \& Klein, W. M. (2006). Experimental manipulations of self-affirmation: A systematic review. Self and Identity, 5, 289-354. 
Mitchell, A. J. (2007). Reluctance to disclose difficult diagnoses: a narrative review comparing communication by psychiatrists and oncologists. Supportive Care in Cancer, 15(7), 819-828.

Mitchell, S. L., Teno, J. M., Kiely, D. K., Shaffer, M. L., Jones, R. N., Prigerson, H. G., . . Hamel, M. B. (2009). The clinical course of advanced dementia. New England Journal of Medicine, 361, 1529-1538.

Morrison, R. S., Wallenstein, S., Natale, D. K., Senzel, R. S., \& Huang, L. L. (2000). “We don't carry that"-failure of pharmacies in predominantly nonwhite neighborhoods to stock opioid analgesics. New England Journal of Medicine, 342, 1023-1026.

Neal, A., Ballard, T., \& Vancouver, J. B. (2017). Dynamic Self-Regulation and Multiple-Goal Pursuit. Annual Review of Organizational Psychology and Organizational Behavior, 4, 401423.

Novak, S., Nemeth, W. C., \& Lawson, K. A. (2004). Trends in medical use and abuse of sustained-release opioid analgesics: a revisit. Pain Medicine, 5, 59-65.

Nutting, P. A. (1994). Competing demands of primary care: a model for the delivery of clinical preventive services. The Journal of family practice, 38, 167-171.

Obermeyer, Z., Makar, M., Abujaber, S., Dominici, F., Block, S., \& Cutler, D. M. (2014). Association between the Medicare hospice benefit and health care utilization and costs for patients with poor-prognosis cancer. JAMA: Journal of the American Medical Association, 312, 1888-1896.

Orehek, E. \& Forest, A. L. (2016). When people serve as means to goals: Implications of a motivational account of close relationships. Current Directions in Psychological Science, 25, 79-84. 
Orehek, E., Forest, A. L., \& Barbaro, N. (in press). A people-as-means approach to interpersonal relationships. Perspectives on Psychological Science.

Orehek, E., Forest, A. L., \& Wingrove, S. (in press). People as means to multiple goals: Implications for interpersonal relationships. Personality and Social Psychology Bulletin.

Orehek, E., Mauro, R., Kruglanski, A. W., \& van der Bles, A. M. (2012). Prioritizing association strength versus value: The influence of self-regulatory modes on means evaluation in single goal and multigoal contexts. Journal of Personality and Social Psychology, 102, 22-31.

Orehek, E., \& Vazeou-Nieuwenhuis, A. (2013). Sequential and concurrent strategies of multiple goal pursuit. Review of General Psychology, 17, 339-349.

Padgett, L., \& Ferrer, R. A. (2015). Palliative care in cancer: Enhancing our view with the science of emotion and decision making. Journal of Palliative Medicine, 18, 479.

Peppercorn, J. M., Smith, T. J., Helft, P. R., Debono, D. J., Berry, S. R., Wollins, D. S., . . . Schnipper, L. E. (2011). American Society of Clinical Oncology Statement: Toward individualized care for patients with advanced cancer. Journal of Clinical Oncology, 29, 755760.

Polman, E. (2010). Information distortion in self-other decision making. Journal of Experimental Social Psychology, 46, 432-435.

Polman, E. (2012a). Effects of self-other decision making on regulatory focus and choice overload. Journal of Personality and Social Psychology, 102, 980.

Polman, E. (2012b). Self-other decision making and loss aversion. Organizational Behavior and Human Decision Processes, 119, 141-150.

Polman, E., \& Emich, K. J. (2011). Decisions for others are more creative than decisions for the self. Personality and Social Psychology Bulletin, 37, 492-501. 
Polman, E., \& Vohs, K. D. (2016). Decision fatigue, choosing for others, and selfconstrual. Social Psychological and Personality Science, 7, 471-478.

Schenker, Y., Crowley-Matoka, M., Dohan, D., Rabow, M. W., Smith, C. B., White, D. B., ... \& Arnold, R. M. (2013). Oncologist factors that influence referrals to subspecialty palliative care clinics. Journal of Oncology Practice, 10, e37-e44.

Shah, J. Y., Friedman, R., \& Kruglanski, A. W. (2002). Forgetting all else: on the antecedents and consequences of goal shielding. Journal of Personality and Social Psychology, 83, 12611280.

Steele, C. M. (1988). The psychology of self-affirmation: Sustaining the integrity of the self. Advances in Experimental Social Psychology, 21, 261-302.

Temel, J. S., Greer, J. A., Muzikansky, A., Gallagher, E. R., Admane, S., Jackson, V. A., . . . Pirl, W. F. (2010). Early palliative care for patients with metastatic non-small-cell lung cancer. New England Journal of Medicine, 363, 733-742.

Vancouver, J. B., Weinhardt, J. M., \& Schmidt, A. M. (2010). A formal, computational theory of multiple-goal pursuit: integrating goal-choice and goal-striving processes. Journal of Applied Psychology, 95, 985-1008.

Vig, E. K., Starks, H., Taylor, J. S., Hopley, E. K., \& Fryer-Edwards, K. (2007). Surviving surrogate decision-making: what helps and hampers the experience of making medical decisions for others. Journal of General Internal Medicine, 22, 1274-1279.

Vohs, K. D., Park, J. K., \& Schmeichel, B. J. (2013). Self-affirmation can enable goal disengagement. Journal of Personality and Social Psychology, 104, 14-27. 
Walter, S. R., Li, L., Dunsmuir, W. T., \& Westbrook, J. I. (2014). Managing competing demands through task-switching and multitasking: a multi-setting observational study of 200 clinicians over 1000 hours. BMJ Qual Saf, 23, 231-241.

Wendler, D., \& Rid, A. (2011). Systematic review: the effect on surrogates of making treatment decisions for others. Annals of Internal Medicine, 154, 336-346.

Wiese, C. H., Bartels, U. E., Marczynska, K., Ruppert, D., Graf, B., \& Hanekopp, G. G. (2009). Quality of out-of-hospital palliative emergency care depends on the expertise of the emergency medical team - a prospective multi-centre analysis. Supportive Care in Cancer, 17, 1499-1506.

Wright, A. A., Zhang, B., \& Ray, A. (2008). Associations between end-of-life discussions, patient mental health, medical care near death, and caregiver bereavement adjustment. JAMA: Journal of the American Medical Association, 300, 1665-1673.

Wrosch, C., Scheier, M. F., Carver, C. S., \& Schulz, R. (2003). The importance of goal disengagement in adaptive self-regulation: When giving up is beneficial. Self and Identity, 2, 1-20.

Wrosch, C., Scheier, M. F., Miller, G. E., Schulz, R., \& Carver, C. S. (2003). Adaptive selfregulation of unattainable goals: Goal disengagement, goal reengagement, and subjective well-being. Personality and Social Psychology Bulletin, 29, 1494-1508.

Yeager, D. S., \& Walton, G. M. (2011). Social-psychological interventions in education: They're not magic. Review of Educational Research, 81, 267-301.

Zhang, Y., Fishbach, A., \& Kruglanski, A. W. (2007). The dilution model: how additional goals undermine the perceived instrumentality of a shared path. Journal of Personality and Social Psychology, 92(3), 389-401. 
Zikmund-Fisher, B. J., Sarr, B., Fagerlin, A., \& Ubel, P. A. (2006). A matter of perspective: choosing for others differs from choosing for yourself in making treatment decisions. Journal of General Internal Medicine, 21(6), 618-622. 